\title{
Review of: "Preloaded D-methionine protects from steady state and impulse noise-induced hearing loss and induces long-term cochlear and endogenous antioxidant effects"
}

\author{
Sima Kianpour Rad
}

Potential competing interests: The author(s) declared that no potential competing interests exist.

This study, entitle "Preloaded D-methionine protects from steady state and impulse noiseinduced hearing loss and induces long-term cochlear and endogenous antioxidant effects" is an authentic work with well-designed experiments and well-writing.

Thanks to the authors for such great work. I am bringing several ideas to this works which are as follows:

One of the interesting part was study of the two types of ototoxicity (impulse and steady state noises) together.

S Mäntysalo and IVuori in 1984 studied the effects on hearing induced by occupational exposure to impulse noise were compared with those induced by exposure to continuous steady state noise. Based on their report, continuous steady state noise has the highest thresholds asymmetrically, in the left ear at $6000 \mathrm{~Hz}$. Longer the duration of exposure to impulse noise the wider the region of the frequencies that showed raised threshold shifts in both ears. Impulse noise seemed to produce permanent threshold shifts at 4000 and $6000 \mathrm{~Hz}$ after a shorter duration of exposure than continuous steady state noise.

So, considering those findings, such different effects of two types of noise on hearing makes them interesting idea to more focus on.

There are also several studies focusing on the effect of the amino acid (D-methionine ) as a strong antioxidant on hearing loss following overexposure to noise (‥ Rewerska, et al, 2013; Yanru Wang et al, 2019; Jhang Ho Pak, 2020) with emphasizing the antioxidant pathway alterations. A double-blind, placebocontrolled Phase 3 clinical trial of oral D-methionine (D-met) indicated reduction of noise-induced hearing loss (NIHL) and tinnitus after oral administration by D-methionine (D-met) and hence, it showed a significant otoprotective activity over an 11 day period.

Therefore, these findings can point to the importance of role of D-methionine on hearing. The study that I am reviewing focused on oxidant pathways which could alter by the two types of ototoxicity and after by treatment with an amino acid. This goal is definitely other compelling part. This work includes the proper time point of induction hear damaging as well as the time points that the 
authors opted to use their treatment. Also, the antioxidant enzymes which they intended to study were well chosen, however, the measurement of ROS produced by the impulse and continues noise could improve the work.

A question arising here in my opinion is the reason of the statement that the authors mentioned in the introduction

“SOD and CAT are demonstrated biomarkers for schizophrenia [28] and 3-methylglutaconic aciduria [29]. Thus, endogenous SOD and CAT enzyme assays may provide feasible and accessible biomarker opportunities to elucidate D-met's optimal protective prophylactic dose and understand how D-met treatment influences the overall antioxidant environment".

How come did they mentioned the activity of SOD and CAT in a mental disorder, such as schizophrenia to conclude that the measurement of such enzymes activity can provide a good opportunities to elucidate Dmet's optimal protective prophylactic dose, etc?.

The discussion is really strong, however, I did not understand why did the CAT activity had no change between the studied groups, while multiple similar studies have shown that D-met could decrease the CAT activity in groups of animal having hear problem.

Totally, the work is enough strong which made it a suitable article to be published in a high ranked journal, like Plos One. 